\title{
Optimization of the flexural rigidity of a concentric pipe used in transmitting rotation for arc-shaped forceps
}

\author{
Satsuya Noda ${ }^{1 *+}\left(0\right.$, Toshio Takayama ${ }^{2+}$ and Toru Omata ${ }^{2+}$
}

\begin{abstract}
Arc-shaped forceps are important in pharyngeal cancer surgery, endoscopic sinus surgery, and single port surgery. Rotating the gripper of an arc-shaped forceps with respect to its shaft axis can improve the manipulability of the forceps. Concentric pipe structures consisting of inner and outer pipes are often used for this purpose. The inner pipe transmits rotations from its proximal end to distal end. However, the shape of the bent inner pipe is generally different from that of the outer pipe, and this causes large friction between the outer and inner pipes. To reduce friction, a plastic tube is often used for the inner pipe. However, since its torsional rigidity is low, this results in a large twist angle. This paper proposes a procedure to fit a metal bent inner pipe to an outer pipe by adjusting the flexural rigidity of the inner pipe with slits to reduce the friction. The procedure minimizes the twist angle between the proximal and distal end of the arc-shaped forceps by reducing the torque required to rotate its metal inner pipe. We develop a $5 \mathrm{~mm}$ forceps with a $3.5 \mathrm{~mm}$ inner pipe. The twist angle is $2.8^{\circ}$ when a load torque of $20 \mathrm{Nmm}$ is applied, which is a typical load torque for suturing, whereas the twist angle of a PEEK (polyetheretherketone) inner pipe is $24.3^{\circ}$ in simulation. Ex vivo experiments show that the required forceps torque is small in simulated clinical scenarios.
\end{abstract}

Keywords: Concentric pipe, Arc-shaped forceps, Single port surgery, Pharyngeal cancer surgery, Flexural rigidity, Slit

\section{Background}

Arc-shaped forceps are important in pharyngeal cancer surgery, endoscopic sinus surgery [1], and single port surgery [2]. The use of a linear-shaped forceps in pharyngeal cancer surgery requires a patient to bend his or her neck backwards and increases the burden on the patient when compared with the surgery performed by an arc-shaped forceps. In single port surgery, the direction of the gripper attached at the end of a linear-shaped forceps is limited to that of the trocar as shown in Fig. 1a. The use of arc-shaped forceps can accommodate the direction of the gripper.

\footnotetext{
*Correspondence: noda.s.ab@m.titech.ac.jp

tSatsuya Noda, Toshio Takayama and Toru Omata contributed equally to this work

${ }^{1}$ Department of Mechano-Micro Engineering, Tokyo Institute of Technology, 4259, Nagatsuta-cho, Midori-ku, Yokohama, Kanagawa 226-8502, Japan

Full list of author information is available at the end of the article
}

The manipulability of a forceps can be improved if its gripper can rotate about its shaft axis [3]. With respect to a linear-shaped forceps, the rotation of the proximal end simply generates the rotation of the distal end as shown in Fig. 1a. However, in an arc-shaped forceps, the rotation of the proximal end moves the position of the distal end as shown in Fig. 1b. There are two types of structures to rotate the distal end, namely multi-joint and concentric pipe structures. Although the multi-joint structure enables the smooth rotation of the end of a forceps, it is complex and consists of several parts.

Surgical robots $[4,5]$ and employ a concentric pipe structure consisting of inner and outer pipes. The arcshaped cannula of the da-Vinci surgical system [6-8] and TANKO (Adachi Industry) [9] also employ a concentric structure for the rotation of the distal end for single port surgery. This method enables a stiffer structure when compared with that in a multi-joint structure.

Generally, the shape of the bent inner pipe is different from that of the outer pipe as shown in Fig. 2. This causes 


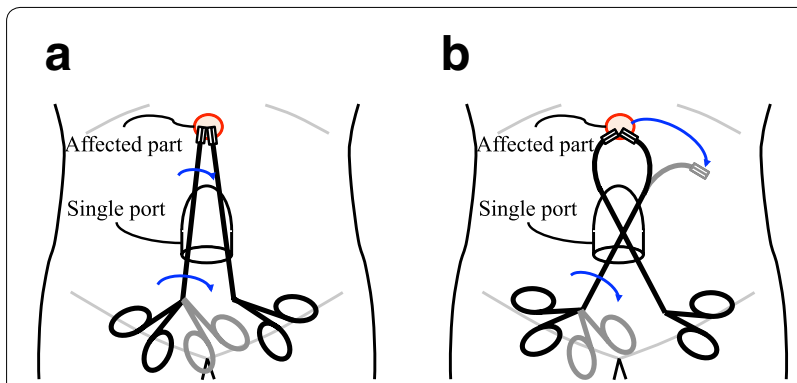

Fig. 1 Linear and arc-shaped forceps. a Linear. b Arc-shaped

contact between the outer and inner pipes, resulting in friction. A load torque as well as the friction increases the torque to rotate the inner pipe. A typical load torque for suturing is $20 \mathrm{Nmm}$ [10]. Using a low friction plastic tube (e.g. PEEK/PTFE composite) for the inner pipe would be valid to reduce friction. However, the Young's modulus of plastic is much lower than that of metal and the torsional rigidity is also low, which results in a large twist angle.

This study proposes a procedure to fit the bent metal inner pipe with respect to the outer pipe such that only the two ends of the pipes are in contact with each other by adjusting the flexural rigidity of the inner pipe with slits as shown in Fig. 2b. Thus, the friction between the pipes can be reduced even if the inner pipe touches the outer pipe in areas other than the ends of the pipes. An inner pipe with optimally adjusted slit intervals can minimize the twist angle.

\section{Adjustment of the flexural rigidity with slits}

Lower flexural rigidity is necessary for an inner pipe. However, torsional rigidity decreases and the twist angle increases as flexural rigidity decreases. Hence, this study proposes an optimization procedure to minimize the twist angle. The ease of manufacturing is also important to develop a practical medical tool, and it is also required to adjust the flexural rigidity within the accuracy of machine tools. In this study, the flexural rigidity of an inner pipe with slits is adjusted.

\section{Equivalent moment of inertia of area}

In a pipe with slits, the moment of inertia of area (an index of flexural rigidity) cannot be calculated from a cross section of the pipes because it varies with respect to the region where the pipe is cut. Therefore, the equivalent moment of inertia of area $I$ and the equivalent modulus of section $Z$ [11] is defined by considering the bending of a pipe with slits with respect to that of a pipe without slits. $I$ and $Z$ can, respectively, be calculated by the following expressions:

$$
\begin{gathered}
I=\frac{F L^{3}}{3 E \delta} \\
Z=\frac{F L}{\sigma_{\max }},
\end{gathered}
$$

where $F$ denotes the force acting on the end, $E$ denotes the Young's modulus of the material of the pipe, $\delta$ denotes the deflection, $\sigma_{\max }$ denotes the maximum tensile stress, and $L$ denotes the length of the cantilever. The finite element method (FEM) is used to analyze $\delta$ and $\sigma_{\max }$. The minimum $L$ is defined as one interval of the slits, but $I$ is calculated using $L=300 \mathrm{~mm}$ to increase accuracy.

Similarly, the equivalent polar moment of inertia of area $I_{p}$ and the equivalent polar modulus of section $Z_{p}$ are given by the following equations:

$$
\begin{aligned}
& I_{p}=\frac{T L}{G \theta} \\
& Z_{p}=\frac{T}{\tau_{\max }},
\end{aligned}
$$

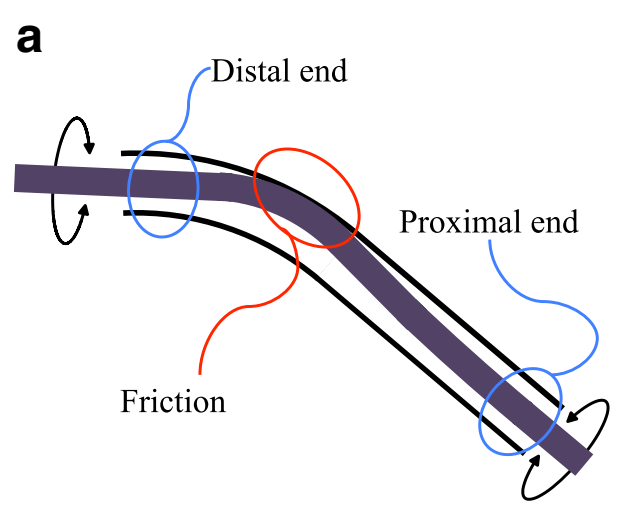

b

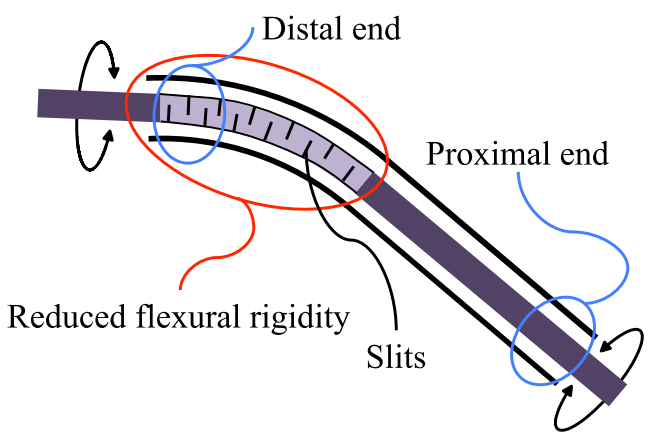

Fig. 2 Inner pipe with insufficient flexibility. a Inner pipe with insufficient flexibility. b Inner pipe with slits exhibiting sufficient flexibility 
where $T$ represents the torsional torque, $G$ represents the shear modulus, $\theta$ represents the torsional angle, $\tau_{\max }$ represents the maximum shearing stress, and $L$ represents the length of the cantilever. The calculations are also performed for $L=300 \mathrm{~mm}$.

\section{Slit pattern}

Various slit patterns exist in shaft couplings and in flexible forceps [12]. In order to enable the ease of manufacturing, two patterns as shown in Fig. 3 are considered, and the pattern that provided low flexural rigidity and high torsional rigidity was selected. The inner pipe has slits in a direction vertical to the paper surface and has the same flexural rigidity in all radial directions. $I$ and $I_{p}$ are calculated by using Mechanica (pro/Engineer). The shape parameters are as follow (see Fig. 3a, c, e):

Outer diameter: $d_{2}=6 \mathrm{~mm}$

Inner diameter: $d_{1}=5 \mathrm{~mm}$

Slit interval $e=1.4 \mathrm{~mm}$

Slit interval $a=1.2,1.6,2.0,2.4$ and $3.0 \mathrm{~mm}$

Slit interval $c=0.3$ and $0.4 \mathrm{~mm}$.

Figure 4 shows the calculation results. The vertical axis is denoted by $I_{p}$, and the horizontal axis is denoted by $I$. It is observed that $I_{p}$ in Pattern $\mathrm{A}$ is higher than that in Pattern B with the same $I$. Thus, Pattern A is selected in this study.

\section{Boundary condition of the ends}

Generally, arc-shaped forceps for pharyngeal cancer surgery or for single port surgery have a curved portion in the distal end region, which is close to the affected part. In contrast, the forceps have a linear portion in the proximal end region in which a handle is attached.

The bending formula is given by the following expression:

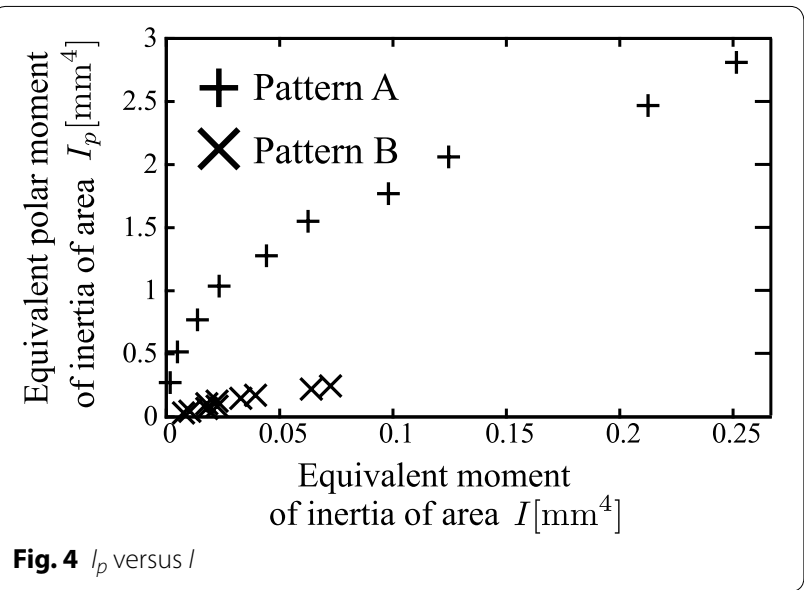

$$
\kappa=-\frac{M}{E I},
$$

where $\kappa$ denotes the curvature, and $M$ denotes the bending moment. Additionally, $M \neq 0$ because $\kappa \neq 0$ at the distal end as shown in Fig. 2b. Thus, two contact points are required to support the non-zero bending moment denoted by $M$. Conversely, $\kappa=0$ at the proximal end and $M=0$. Therefore, a single contact point is sufficient.

\section{Procedure to optimally adjust the flexural rigidity}

The outline of the procedure proposed in this study is as follows:

Step 1: Develop the database of $I, I_{p}, Z$, and $Z_{p}$ by varying the parameters for the slit.

Step 2: Set a target line, and calculate the curvature along the line.
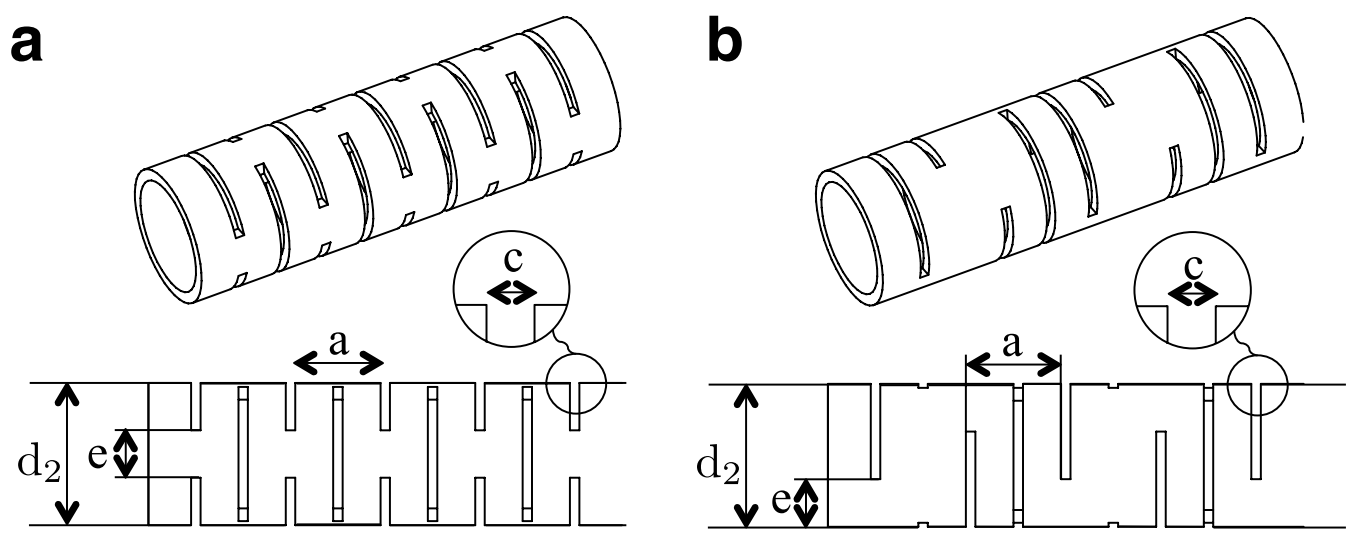

Fig. 3 Slit patterns for an inner pipe. a Pattern A. b Pattern B 
Step 3: Calculate $I$, and determine the range of $f_{H}$, which corresponds to the contact force acting on the proximal end. Define the range as $f_{\min } \leq f_{H} \leq f_{\max }$.

Step 4: Determine $f_{H}$ that minimizes the twist angle of the inner pipe $\phi$ in the range $f_{\min } \leq f_{H} \leq f_{\max }$. Calculate $\phi$ for each sampled $f_{H}$ as follows;

Step 4.1: Discretize the distribution of $I$.

Step 4.2: Confirm that the stress on the inner pipe is lower than the maximum permitted stress and that the error between the target and designed lines is less than the given allowable error. If the candidate does not meet these conditions, then it is discarded.

Step 5: Select $f_{H}$ that minimizes $\phi$. If no candidates remain, then return to Step 2 and modify the target line. A solution is more likely to exist if the curvature of the forceps is reduced.

Each step can be described in detail as follows.

\section{Step 1}

The following values were selected for the slit parameters of the inner pipe. Their intervals are the smallest possible for usual machine tools.

$$
\begin{aligned}
& a=1.2,1.6,2.0,2.4,3.0 \mathrm{~mm} \\
& e=1.0,1.4,1.8 \mathrm{~mm} \\
& c=0.3,0.4,0.5 \mathrm{~mm}
\end{aligned}
$$

Calculate $I$ for all combinations of the above cut parameters and sort the calculations in ascending order $\left(I_{1}<I_{2}<\cdots<I_{45}\right)$. This is defined as the database of $I$. Similarly, $I_{p}, Z$ and $Z_{p}$ are calculated corresponding to the I database.

\section{Step 2}

This step provided a target line as shown in Fig. 5. Let $L$ be the total length of the target line, and let $x_{t}(s)$ and $y_{t}(s)$ denote the position of the target line where $s$ is the arc length from the distal end. The curvature $\kappa(s)$ is given as follows:

$$
\kappa(s)=\sqrt{\left(x_{t}^{\prime \prime}(s)\right)^{2}+\left(y_{t}^{\prime \prime}(s)\right)^{2}}
$$

where" denotes the second derivative with respect to $s$.

\section{Step 3}

This step calculates $I$ and determines the range of $f_{H}$. The relationship between the bending moment of the pipe $M_{z}(s)$ and $f_{H}$ is as follows:

$$
M_{z}(s)=-D(s) f_{H},
$$

where

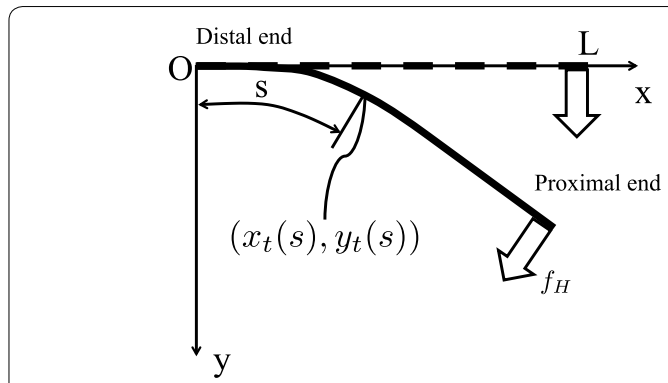

Fig. 5 Coordinate of the bent inner pipe

$$
D(s) \equiv\left[\begin{array}{c}
x_{t}(L)-x_{t}(s) \\
y_{t}(L)-y_{t}(s)
\end{array}\right] \otimes\left[\begin{array}{c}
-y_{t}^{\prime}(L) \\
x_{t}^{\prime}(L)
\end{array}\right],
$$

' denotes the derivative with respect to $s$, and $\otimes$ is a scalar operation for $2-\mathrm{D}$ vectors defined as follows:

$$
\left[\begin{array}{l}
a \\
b
\end{array}\right] \otimes\left[\begin{array}{l}
c \\
d
\end{array}\right]=a d-b c .
$$

Let $I(s)$ be the moment of inertia of area at $s$. The relationship between $I(s)$ and $f_{H}$ is given by

$$
I(s)=h(s) f_{H},
$$

where

$$
h(s)=\frac{D(s)}{E \kappa(s)} .
$$

It is noted that $I(s)$ cannot be lower than the minimum value in the database $I_{\min }$. Therefore, $f_{\min }$, which corresponds to the minimum value of $f_{H}$ must satisfy the following expression:

$$
f_{H} \geq f_{\min }=\max \frac{I_{\min }}{h(s)}=\frac{I_{\min }}{\min h(s)} .
$$

Note 1: If the target line has linear segments, then $\kappa(s)=0$ and $I(s) \rightarrow \infty$ from Eqs. (10) and (11). This type of a target line is impractical and it must be modified. A pipe with no slit has the largest $I(s)$, which is denoted by $I_{\max }$. Hence, $I(s)$ must be replaced by $I_{\max }$ in portions where $I(s) \geq I_{\max }$. Since $I(s)$ is reduced, $f_{\min }$ is also reduced so that the proximal end of the pipe is as close as possible to that of the target line.

Then, $f_{\max }$ is set as the maximum value of $f_{H}$. For example, $f_{\max }=100 f_{\min }$ in simulation section. In $f_{\min } \leq f_{H} \leq f_{\max }$, the $f_{H}$ that minimizes $\phi$ is determined.

Figure 6 shows the forces and moments acting on the inner pipe, which is in contact with the outer pipe only at its ends. The following symbols are defined:

$T_{m}$ : axial torque acting on the inner pipe between the proximal and distal ends

$T_{\text {OUT: }}$ output torque at the distal end of the inner pipe $f_{z 0}$ : contact force in the $\mathrm{z}$ direction 


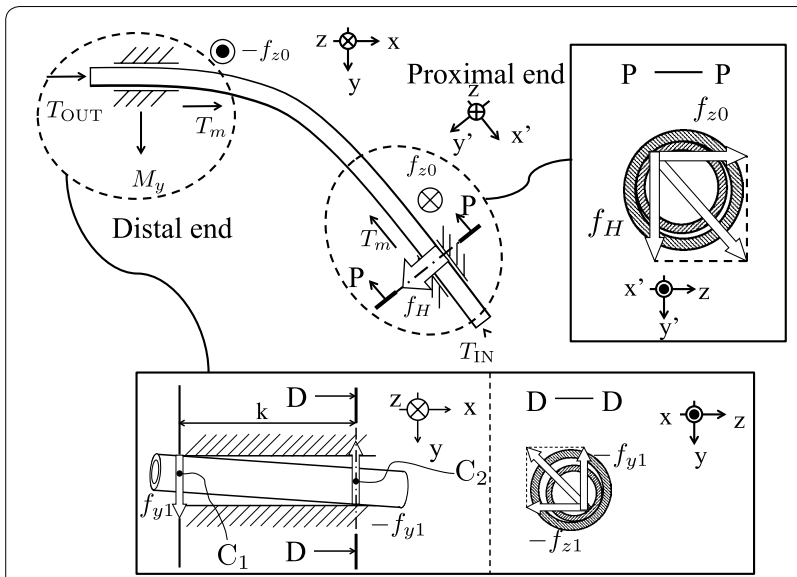

Fig. 6 Forces and torques acting on the inner pipe

$C_{1}$ and $C_{2}$ : two contact points at the distal end $k$ : distance between $C_{1}$ and $C_{2}$

$f_{y 1}$ and $-f_{y 1}$ : couple of forces acting at $C_{1}$ and $C_{2}$ in the y direction

$f_{z 1}$ and $-f_{z 1}$ : couple of forces acting at $C_{1}$ and $C_{2}$ in the $\mathrm{z}$ direction

$M_{y}$ moment about the Y-axis generated by $f_{z 1}$ and $-f_{z 1}$.

The equilibrium equation of the moment on the $x-y$ plane is given as follows:

$$
\begin{aligned}
{\left[\begin{array}{l}
0 \\
0
\end{array}\right]=} & T_{m}\left[\begin{array}{l}
1 \\
0
\end{array}\right]-T_{m}\left[\begin{array}{c}
x_{t}^{\prime}(L) \\
y_{t}^{\prime}(L)
\end{array}\right] \\
& +M_{y}\left[\begin{array}{l}
0 \\
1
\end{array}\right]+f_{z 0}\left[\begin{array}{c}
y_{t}(L) \\
-x_{t}(L)
\end{array}\right] .
\end{aligned}
$$

This results in the following expression:

$$
T_{m}=\frac{T_{\text {OUT }}+\gamma \sqrt{\alpha^{2}\left(1-\beta^{2} \gamma^{2}\right) k^{2} f_{H}^{2}+\beta^{2} T_{\text {OUT }}^{2}}}{1-\beta^{2} \gamma^{2}},
$$

where $\alpha, \beta$, and $\gamma$ are constants given by

$$
\begin{aligned}
& \alpha=\frac{D(0)}{k} \\
& \beta=y_{t}^{\prime}(L)-\frac{x_{t}(L)}{y_{t}(L)}\left(1-x_{t}^{\prime}(L)\right) \\
& \gamma=\frac{\mu d_{2}}{k} .
\end{aligned}
$$

Equation (14) can be obtained as follows. From Eq. (13), we have:

$$
\begin{aligned}
& f_{z 0}=-\frac{1-x_{t}^{\prime}(L)}{y_{t}(L)} T_{m} \\
& M_{y}=\left[y_{t}^{\prime}(L)-\frac{x_{t}(L)}{y_{t}(L)}\left(1-x_{t}^{\prime}(L)\right)\right] T_{m} .
\end{aligned}
$$

Since $x_{t}(L) \gg k$, it can be assumed that $f_{y 1}$ and $-f_{y 1}$ generate $M_{z}(0)$, Similarly, since $y_{t}(L) \gg k$, it is assumed that $f_{z 1}$ and $-f_{z 1}$ generate $M_{y}$. Hence,

$$
\begin{gathered}
f_{\mathrm{y} 1}=\frac{M_{z}(0)}{k}=\alpha f_{H} \\
f_{\mathrm{z} 1}=\frac{M_{y}}{k}=\frac{\beta}{k} T_{m},
\end{gathered}
$$

Subtracting the friction acting at the distal end from $T_{m}$ results in $T_{\text {OUT }}$. Hence,

$$
T_{\mathrm{OUT}}=T_{m}-2 \frac{\mu d_{2}}{2} \sqrt{f_{\mathrm{y} 1}^{2}+f_{\mathrm{z} 1}^{2}},
$$

where $\mu$ denotes the coefficient of friction. Eq. (14) can be obtained by Eqs. (18-22).

The angle of torsion $\phi$ can be calculated by the following expression:

$$
\phi=\int_{0}^{L} \frac{T_{m}}{G I_{p}(s)} d s
$$

where $I_{p}(s)$ denotes the polar moment of inertia of area at $s$.

$f_{H}$ is sampled in the range $f_{\min } \leq f_{H} \leq f_{\max }$ to evaluate the angle $\phi$ becuase the monotonicity of $\phi$ in Eq. (23) cannot be determined as the value of $f_{H}$ increases.

Note 2: $I_{p}$ of the denominator monotonically increases as the value of $I$ increases as shown in Fig. 4. From Eq. (10), $I$ weakly increases as the value of $f_{H}$ increases. Thus, $I_{p}$ weakly increases as the value of $f_{H}$ increases. $T_{m}$ of the numerator also monotonically increases as the value of $f_{H}$ increases.

\section{Step 4}

The following are calculated for each $f_{H_{j}}=f_{\text {min }}+j \Delta h$ where $\Delta h=\left(f_{\max }-f_{\min }\right) / N$ and $j=0, \ldots, N$.

\section{Step 4.1}

Discretize $I$ (quantization) and $s$ (sampling).

It is assumed that $I(s)$ is given as shown in Fig. 7. $I$ is discretized by using the database of $I$ as illustrated in Fig. 7 .

1. In $I_{1}, \ldots, I_{45}$ of the database, the set of $I_{i}$ that satisfies $\min I(s) \leq I_{i} \leq \max I(s)$ is determined. It is assumed that $I_{a}<I_{a+1}<\cdots<I_{b}$ are such $I_{i}$.

2. Let $A_{1}, A_{2}, \ldots$, and $A_{\mathrm{n}}$ denote the intersection points between $I_{a}<I_{a+1}<\cdots<I_{b}$ and $I(s)$ in the ascending order of $s$.

3. Let $\hat{I}_{1}, \hat{I}_{2}, \ldots$, and $\hat{I}_{n}$ denote the values of $I(s)$ at points $A_{1}, A_{2}, \ldots$, and $A_{\mathrm{n}}$, respectively.

4. $B_{i}$ be the middle point between $A_{i}$ and $A_{i+1}$ on the $s$ axis, where $i=1, \ldots, n-1$. 


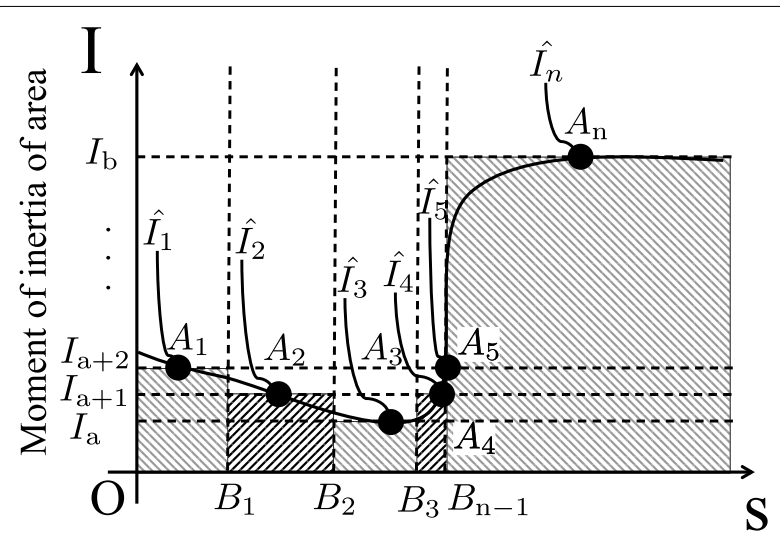

Arc-length from the distal end

Fig. 7 Sampling of /(s) with respect to $s$

5. For each interval $O B_{1}, B_{1} B_{2}, \ldots$, and $B_{n-1} A_{n}, I(s)$ can be discretized as $\hat{I}_{1}, \hat{I}_{2}, \ldots$, and $\hat{I}_{n}$, respectively.

Note 3: The discretization of $I(s)$ could cause an error between the proximal end positions of the designed and target lines. Therefore, $f_{H}$ is modified so that they are as close as possible.

\section{Step 4.2}

The following three procedures are executed for confirmation:

\section{Simple stress confirmation}

Let $\sigma_{\text {net }}$ be the maximum stress when both the bending and torsional loads are applied. $\sigma_{\text {net }}$ must be smaller than the proof stress $\sigma_{\text {proof }}$ of the material (typically, $0.2 \%$ proof stress). Accurate calculation of the stress by using FEM (CPU: Core i7 3.4 GHz) requires a large calculation time exceeding an hour. Therefore, the following prescreening is performed using a simple criterion. Let $\sigma_{s}$ and $\tau_{s}$ denote the maximum bending and the maximum torsional stresses, respectively.

$$
\begin{aligned}
\sigma_{s} & =\operatorname{Max} \frac{M_{z}(s)}{Z(s)} \\
\tau_{s} & =\operatorname{Max} \frac{T_{m}}{Z_{p}(s)},
\end{aligned}
$$

where $Z(s)$ and $Z_{p}(s)$ denote the modulus of section and the polar modulus of section at $s$, respectively.

$\sigma_{\text {net }}$ should be calculated. However, the slits of the inner pipe makes the calculation of $\sigma_{\text {net }}$ from $\sigma_{s}$ and $\tau_{s}$ difficult. In many cases of this study, $\sigma_{s} \gg \tau_{s}$. Moreover, the stress concentration points of the bending load are different from those of the torsional load as will be described in the simulation section. Therefore, this step regards that $\sigma_{\text {net }} \approx \sigma_{s}$ and excludes the $j$ th candidate $f_{H j}$ if $\sigma_{s}>\sigma_{\text {proof }}$.

\section{Error}

The maximum error between the target and the designed lines is denoted by $\varepsilon$. It is confirmed that the calculated value is smaller than a given allowable error $\bar{\varepsilon}$. If $\varepsilon<\bar{\varepsilon}$, then the procedure moves to the next step. If $\varepsilon>\bar{\varepsilon}$, then the sampling intervals are randomly selected and modified by several millimeters, and the procedure returns to Step 4.2 (1). It should be noted that this random search is practical because the number of the sampling intervals is expected to be less than 10 . If $\varepsilon>\bar{\varepsilon}$ after this procedure, then $f_{H_{j}}$ is excluded.

\section{Final stress confirmation}

$\sigma_{\text {net }}$ is calculated by using FEM. The $j$ th candidate is excluded, if $\sigma_{\text {net }}>\sigma_{\text {proof }}$.

\section{Step 5}

The final step involves selecting $f_{H_{j}}$ that minimizes $\phi . \phi$ of each candidate is obtained by Eq. (23). If no candidates of $f_{H_{j}}$ remain, then the procedure returns to Step 2 and modifies the target line by reducing its curvature.

\section{Simulation and experiment}

\section{Simulation of $8 \mathrm{~mm}$ forceps}

First, simulations for an arc-shaped forceps for single port surgery are performed. Its shape is given by the following expression:

$$
\begin{aligned}
{\left[\begin{array}{l}
x_{t}(s) \\
y_{t}(s)
\end{array}\right]=} & R_{0}\left[\begin{array}{c}
\sin \frac{s}{R_{0}} \\
1-\cos \frac{s}{R_{0}}
\end{array}\right]\left(0 \leq s \leq R_{0} \theta\right) \\
{\left[\begin{array}{l}
x_{t}(s) \\
y_{t}(s)
\end{array}\right]=} & R_{0}\left[\begin{array}{c}
\sin \theta \\
1-\cos \theta
\end{array}\right] \\
& +\left(s-R_{0} \theta\right)\left[\begin{array}{l}
\cos \theta \\
\sin \theta
\end{array}\right] \\
& \left(R_{0} \theta \leq s \leq L\right),
\end{aligned}
$$

where $R_{0}$ denotes the radius of the circular arc, and $\theta$ denotes the central angle of the circular arc. Given the da-Vinci arc-shaped cannula [6], the parameters are set as $R_{0}=120 \mathrm{~mm}, \theta=60^{\circ}$, and $L=300 \mathrm{~mm}$.

The material assumed in the FEM calculation is titanium (Ti-6Al-4V, Young's modulus: $113 \mathrm{GPa}, 0.2 \%$ proof stress: $828 \mathrm{MPa}$ ). The outer and inner diameters of the inner pipe are 6 and $5 \mathrm{~mm}$, respectively. These dimensions were selected for an $8 \mathrm{~mm}$ forceps. From Eq. (12), the search range is obtained as $9.94 \times 10^{-3} \leq f_{H} \leq 9.94 \times 10^{-1} \mathrm{~N}$. However, because $\sigma_{\text {net }}>\sigma_{\text {proof }}$ when $f_{H}>1.5 \times 10^{-1} \mathrm{~N}$, it is reduced to $9.94 \times 10^{-3} \leq f_{H} \leq 1.5 \times 10^{-1} \mathrm{~N}$. It is assumed that 
$\mu=0.3, k=10 \mathrm{~mm}$ and $T_{O U T}=20.0 \mathrm{Nmm}$. The value of $T_{\text {OUT }}$ is determined by referring to the suture task in [10].

Table 1 shows the calculation result of $\phi$ for each candidate. From Table 1, $\phi$ is observed as the smallest when $f_{H}=0.13 \mathrm{~N}$. Table 2 shows the slit parameters and errors. Three different slits are cut in the intervals $O B_{1}, B_{1} B_{2}$, and $B_{2} B_{3}$. In Step $4.2(1), \sigma_{s}=622 \mathrm{MPa}$ and $\tau_{s}=132 \mathrm{MPa}$, and in Step $4.2(3), \sigma_{\text {net }}=678 \mathrm{MPa}$, which is smaller than the $0.2 \%$ proof stress of $828 \mathrm{MPa}$.

Figure 8(1) shows the bent shape of the inner pipe. For purposes of comparison, Fig. 8(2) shows the line with slits cut uniformly, and (3) shows the line with no slits. In (3), $f_{H}$ is such that the maximum stress $\sigma_{s}$ is equal to the proof stress $\sigma_{\text {proof }}$. Line (1) is close to the target line $(\varepsilon=$ $1.25 \mathrm{~mm}$ ) whereas Lines (2) and (3) are not close to the target line $(\varepsilon=16.54,161.9 \mathrm{~mm})$. From Eq. (23), the twist angle is $\phi=4.30^{\circ}$ in (1).

Note 4: The twist angle can also be obtained from the FEM simulation. It is $\phi=4.18^{\circ}$ and close to the twist angle from Eq. (23)

\section{Simulation of $5 \mathrm{~mm}$ forceps}

An arc-shaped forceps with diameter of $5 \mathrm{~mm}$ is simulated. Figure 9 shows the target line with the shape parameters $R_{0}=350 \mathrm{~mm}, \theta=30^{\circ}$ and $L=300 \mathrm{~mm}$. This line is determined by referring to a forceps for pharyngeal cancer surgery that is practically used and a forceps for single port surgery (for example, Type KTY1 of ADACHI-TANKO forceps [13]).

Strong surgical stainless (SUS630, Young's modulus: $205 \mathrm{GPa}, 0.2 \%$ proof stress : $1175 \mathrm{MPa}$ ) was selected as the material for this forceps. From Eq. (12), the search range is determined as $7.23 \times 10^{-2} \leq f_{H} \leq 7.23 \mathrm{~N}$. However, because $\sigma>\sigma_{\text {proof }}$ when $f_{H}>3.0 \times 10^{-1} \mathrm{~N}$, the search range is reduced to $f_{H_{j}}$ in $7.23 \times 10^{-2} \leq f_{H} \leq 3.0 \times 10^{-1}$ $\mathrm{N}$. The other experimental parameters are the same as those discussed in Section Simulation of $8 \mathrm{~mm}$ forceps.

Table 3 shows the calculated results of $\phi$. The value of $\phi$ is the smallest for the 5th candidate, and it is $2.93^{\circ}$. Table 4 shows the slit parameters and errors, and it is observed that two different slits are cut in the interval $O B_{1}$ and $B_{1} B_{2}$. Figure 9 shows the line of the inner pipe

Table 1 Candidate of $f_{H}$ for the $6 \mathrm{~mm}$ inner pipe

\begin{tabular}{llll}
\hline Candidate No. & $\boldsymbol{f}_{\boldsymbol{H}}(\mathbf{N})$ & $\boldsymbol{\phi}\left(^{\circ}\right)$ & $\boldsymbol{\varepsilon}(\mathbf{m m})$ \\
\hline 1st candidate & 0.03 & 7.60 & 0.59 \\
2nd candidate & 0.05 & 5.48 & 0.39 \\
3rd candidate & 0.08 & 5.31 & 1.42 \\
4th candidate & 0.10 & 4.46 & 2.25 \\
5th candidate & 0.13 & 4.30 & 1.25 \\
6th candidate & 0.15 & 4.33 & 0.61 \\
\hline
\end{tabular}

Table 2 Slit parameters and errors of the $6 \mathrm{~mm}$ inner pipe of the 5 th candidate

\begin{tabular}{cc}
\hline $\mathrm{OB}_{1}(\mathrm{~mm})$ & 38 \\
$\mathrm{a}(\mathrm{mm})$ & 1.6 \\
$\mathrm{e}(\mathrm{mm})$ & 1.8 \\
$\mathrm{C}(\mathrm{mm})$ & 0.3 \\
$\mathrm{~B}_{1} \mathrm{~B}_{2}(\mathrm{~mm})$ & 31 \\
$\mathrm{a}(\mathrm{mm})$ & 2.0 \\
$\mathrm{e}(\mathrm{mm})$ & 1.0 \\
$\mathrm{C}(\mathrm{mm})$ & 0.4 \\
$\mathrm{~B}_{2} \mathrm{~B}_{3}(\mathrm{~mm})$ & 57 \\
$\mathrm{a}(\mathrm{mm})$ & 2.0 \\
$\mathrm{e}(\mathrm{mm})$ & 1.4 \\
$\mathrm{C}(\mathrm{mm})$ & 0.5 \\
$\varepsilon(\mathrm{mm})$ & 1.25 \\
\hline
\end{tabular}

obtained by simulation. The designed line is close to the target line $(\varepsilon=1.45 \mathrm{~mm})$.

Table 5 lists the details of the calculation for the 5th candidate. Although the sum of $\sigma_{s}$ and $\tau_{s}$ exceeds $\sigma_{\text {proof }}$ in Step 4.2 (1), $\sigma_{\text {net }}=1169 \mathrm{MPa}$ in Step 4.2 (3), which is smaller than $\sigma_{\text {proof }}$. In any slit, the stress concentration point of the bending load is different from that of the torsional load. Figure 10 shows an example of the FEM results. In addition, the twist angle of a PEEK pipe without slits (Young's modulus: $4.1 \mathrm{GPa}, 0.2 \%$ Proof stress: $163 \mathrm{MPa}$ ) was computed for comparison. It was $24.3^{\circ}$ even when no friction is assumed, which is much larger than $2.93^{\circ}$.

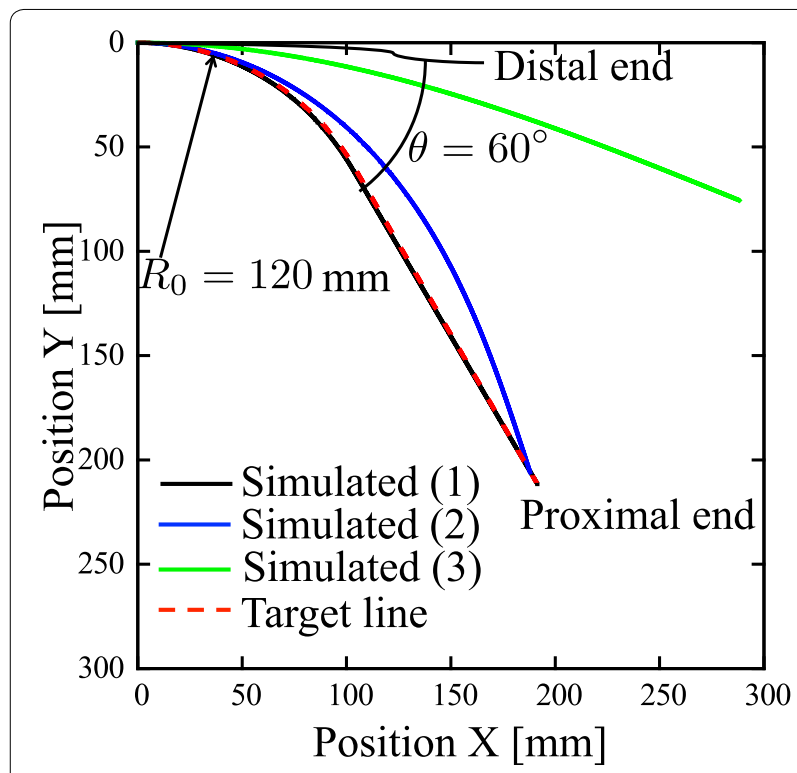

Fig. 8 Simulation results for the $6 \mathrm{~mm}$ inner pipe 


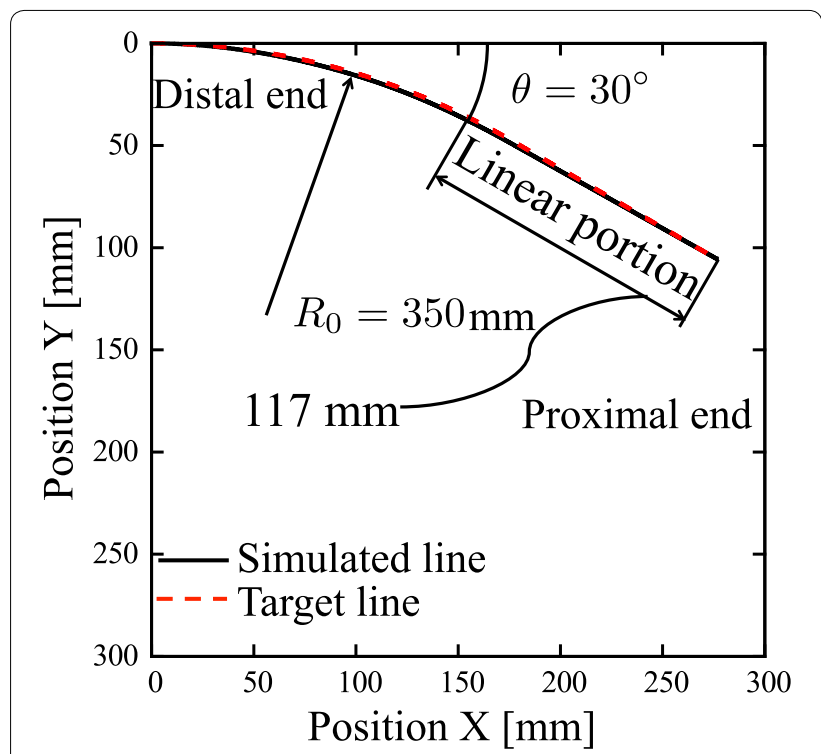

Fig. 9 Simulation results of the $3.5 \mathrm{~mm}$ inner pipe

Table 3 Candidate of $f_{H}$ for the $\mathbf{3 . 5} \mathbf{~ m m}$ inner pipe

\begin{tabular}{llll}
\hline Candidate No. & $\boldsymbol{f}_{\boldsymbol{H}}(\mathbf{N})$ & $\boldsymbol{\phi}\left(^{\circ}\right)$ & $\boldsymbol{\varepsilon}(\mathbf{m m})$ \\
\hline 1st candidate & 0.10 & 4.41 & 1.08 \\
2nd candidate & 0.15 & 3.48 & 3.94 \\
3rd candidate & 0.20 & 3.25 & 0.85 \\
4th candidate & 0.25 & 2.99 & 1.63 \\
5th candidate & 0.30 & 2.93 & 1.45 \\
\hline
\end{tabular}

Table 4 Slit parameters and errors for the $\mathbf{3 . 5} \mathbf{~ m m}$ inner pipe of the 5th candidate

\begin{tabular}{cl}
\hline $\mathrm{OB}_{1}(\mathrm{~mm})$ & 102 \\
$\mathrm{a}(\mathrm{mm})$ & 1.6 \\
$\mathrm{e}(\mathrm{mm})$ & 1.4 \\
$\mathrm{C}(\mathrm{mm})$ & 0.3 \\
$\mathrm{~B}_{1} \mathrm{~B}_{2}(\mathrm{~mm})$ & 81 \\
$\mathrm{a}(\mathrm{mm})$ & 1.6 \\
$\mathrm{e}(\mathrm{mm})$ & 1.4 \\
$\mathrm{C}(\mathrm{mm})$ & 0.4 \\
$\varepsilon(\mathrm{mm})$ & 1.50 \\
\hline
\end{tabular}

Similarly, Table 5 lists the results when the material of the inner pipe was replaced with Ti-6Al-4V and the usual stainless steel SUS304. Ti-6Al-4V has low Young's modulus and similar proof stress as SUS630. This results in a lower $\sigma_{\text {net }}$ but larger twist angle: $\phi=4.93^{\circ}$. However, the twist angle is still much smaller than that of the PEEK pipe.
Table 5 Specification of the 5 th candidate

\begin{tabular}{llll}
\hline Material & SUS630 & Ti-6Al-4V & SUS304 \\
\hline$E(\mathrm{GPa})$ & 205 & 113 & 192 \\
$\sigma_{\text {proof }}(\mathrm{MPa})$ & 1175 & 828 & 205 \\
$\sigma_{S}(\mathrm{MPa})$ & 1144 & 631 & 1071 \\
$\tau_{S}(\mathrm{MPa})$ & 157 & 139 & 155 \\
$\sigma_{\text {net }}(\mathrm{MPa})$ & 1166 & 709 & 1131 \\
$\phi\left(^{\circ}\right)$ & 2.93 & 4.93 & 3.12 \\
\hline
\end{tabular}

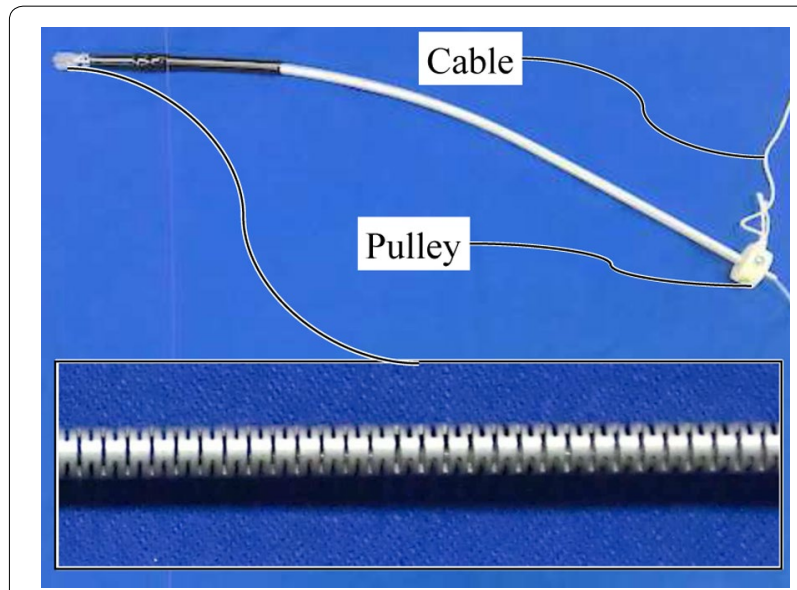

Fig. 10 The maximum stress. Color bar shows Von Mises stress

\section{Development of $5 \mathrm{~mm}$ arc-shaped forceps} and measurement of rotation torque and twist angle To confirm the result of the SUS630 inner pipe, SUS304 was selected to develop the forceps for the experiments owing to its immediate availability. The Young's modulus of SUS304 is $192 \mathrm{GPa}$, and its $0.2 \%$ proof stress is 205 $\mathrm{MPa}$. Although the maximum stress exceeds the proof stress of SUS304, it does not mean that metal fatigue occurs immediately. It can withstand for a certain period. Figure 11 shows the developed forceps.

The torque to rotate the distal end of the inner pipe when no load is applied is measured. An inner pipe with no slits is also prepared for comparison purposes. A handle (material: ABS (acrylonitrile-butadiene-styrene), diameter: $20 \mathrm{~mm}$ ) with a cable was installed on the proximal end, and a force gage measured the tension force. The experiments did not involve the use of bearings. The torque to rotate the distal end is $5.1 \mathrm{Nmm}$, whereas that of the no-slit pipe is $37.0 \mathrm{Nmm}$. The distal end of the developed pipe can be rotated manually, whereas that of the no-slit pipe cannot be rotated manually. The twist angle is $2.8^{\circ}$ when a load torque of $20 \mathrm{Nmm}$ is applied. The result is as small as the simulation result and considerably less than $24.3^{\circ}$. 


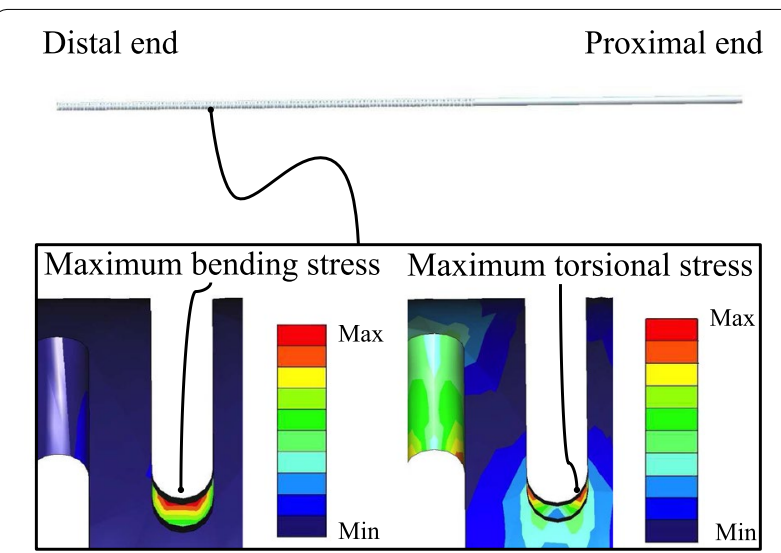

Fig. 11 Developed forceps with the $3.5 \mathrm{~mm}$ inner pipe

\section{Ex vivo experiment}

We performed ex vivo experiments to confirm that the rotation torque does not increase in more realistic conditions. Since the inner diameter of the outer pipe was $4.0 \mathrm{~mm}$ and the outer diameter of the inner pipe was $3.5 \mathrm{~mm}$, a PTFE (polytetrafluoroethylene) tape of $0.15 \mathrm{~mm}$ thickness was attached to the distal end of the inner pipe to prevent organic materials from entering the space between the inner and outer pipes. The maximum clearance between the outer and inner pipes is $0.2 \mathrm{~mm}$, while the minimum slit width is $0.3 \mathrm{~mm}$. Therefore, if any organic material gets into the space, it would be smaller than $0.2 \mathrm{~mm}$. It was expected that such small particles would not clog the slits.

In the ex vivo experiment, chicken liver, minced pork meat and porcine blood were used. In the chicken liver experiment, the distal end of the forceps was inserted into the slit cut of the liver, as shown in Fig. 12a, so that organic materials could fill the space of the forceps. In the minced pork meat experiment, water was added to produce a liquid condition, as shown in Fig. 12b. Figure 12c shows the image of minced meat, as observed with a microscope. Particles with a diameter of approximately $0.2 \mathrm{~mm}$ are seen. Figure $12 \mathrm{~d}$ shows the ex vivo experiment involving the insertion of forceps into the blood. Approximately $30 \mathrm{~mm}$ length of the slit distal side of the forceps was inserted into the blood. Because the purchased blood included $10 \%$ anticoagulant (3.23\% sodium citrate solution), $2 \%$ coagulant ( $2 \%$ calcium chloride solution) was added. The distal end of the forceps was rotated two revolutions every $30 \mathrm{~s}$, for a duration of $4 \mathrm{~h}$. Moreover, the blood was poured into the outer pipe and the inner pipe was inserted into the outer pipe, considering the case of blood invasion into the forceps.

After the ex vivo experiments, we measured the torque required to rotate the inner pipe when no load was applied to the distal end. In the chicken liver experiment, it increased from 5.1 to $5.3 \mathrm{Nmm}$, in the minced pork meat experiment, it increased from 5.1 to $5.4 \mathrm{Nmm}$, and in the porcine blood experiment, it decreased from 5.1 to $3.8 \mathrm{Nmm}$. Moreover, $2 \mathrm{~h}$ after the insertion of blood into the outer pipe, it increased from 5.1 to $5.3 \mathrm{Nmm}$. These increases are small.

\section{Discussion}

\section{Ex vivo experiment}

In the experiments involving the forceps, the twist angle is $2.8^{\circ}$ when a load torque of $20 \mathrm{Nmm}$ is applied. In the ex vivo experiments, the maximum increase of the torque required to rotate the inner pipe is only $0.3 \mathrm{Nmm}$. Therefore, the increase of the twist angle can be expected to be small.

In the chicken liver and minced pork experiments, the small increase in the torque was resulted from the little invasion of organic material into the forceps. In the experiments of the forceps insertion to the blood, the decrease in the torque was caused by fluid lubrication of the blood. In the experiments of the blood impregnation into the outer pipe, the small increase in the torque resulted from gel-like blood clots between the outer and inner pipes. Blood coagulates and forms blood clots, which are fragile gel materials unless they dry out. The blood clots did not dry out because the inner pipe was not exposed to the atmosphere. For this reason, we consider that the blood clot remains as a gel material and fixing does not arise.

\section{Other methods to reduce friction}

The use of PTFE coating is a valid method to reduce friction. The coefficient of friction is typically 0.1 . However, the PTFE coating has low wear resistance and therefore cannot be used on metal-metal contact [14]. In reference [15], PTFE coating is used under low contact pressure.

\section{Sterilization}

The developed forceps should be sterilizable because its metal slit is relatively expensive. If the forceps can be disassembled easily, each part of the forceps can be sterilized. Figure 13 shows the structure of the forceps that can be disassembled. The wire unit that transmits the opening and closing motion of the gripper is detachable from the inner pipe. The disassembly procedure of the forceps is as follows.

1. The wire unit is pulled from the inner pipe from its distal end.

2. The inner pipe is pulled from the outer pipe from its proximal end.

Note: The inner pipe cannot be pulled from the distal end of the outer pipe because the proximal side of the inner 

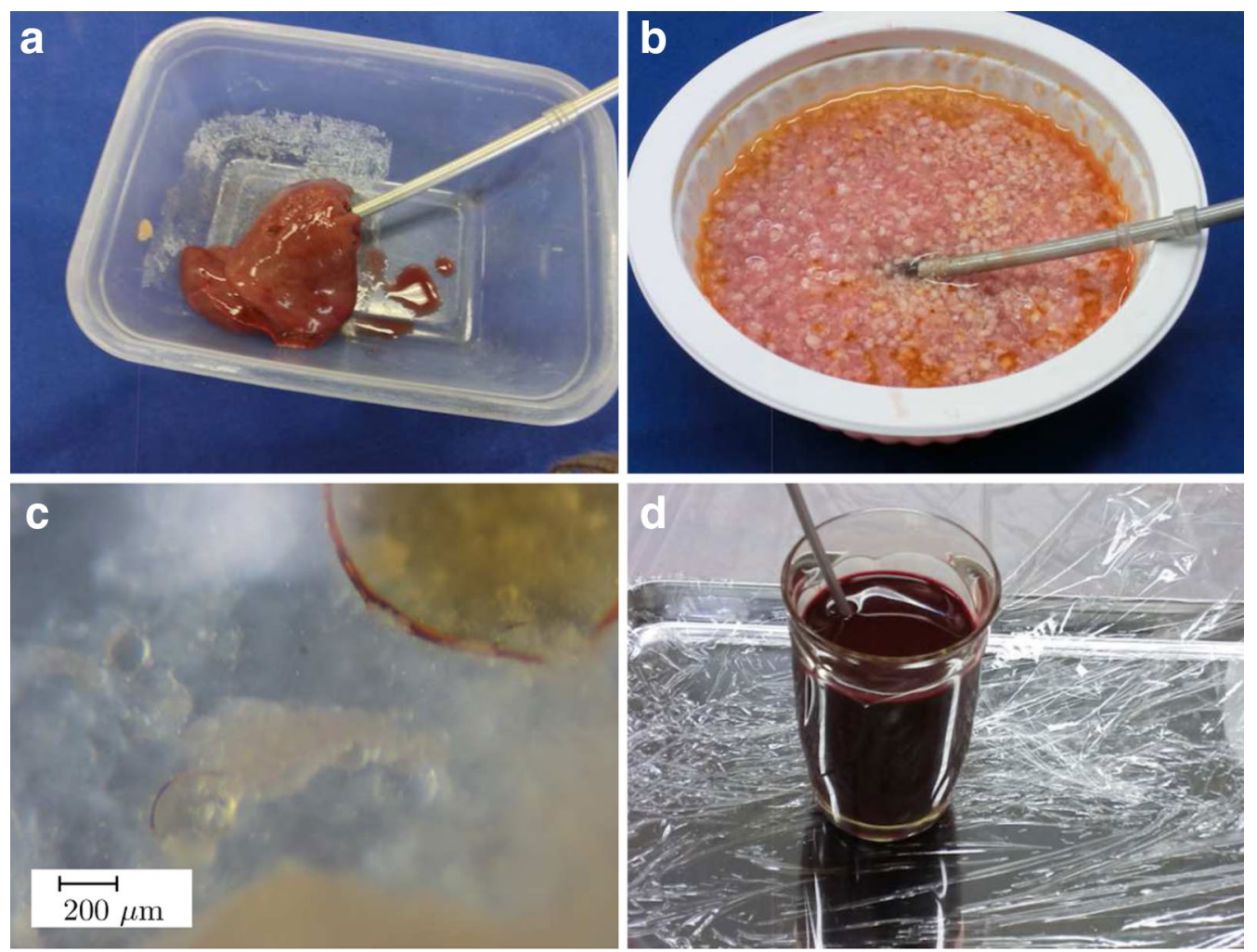

Fig. 12 Ex vivo experiment set up and minced meat. a Chicken liver. b Minced porkand water. c Minced meat asobservedwith a microscope. d Porcine blood

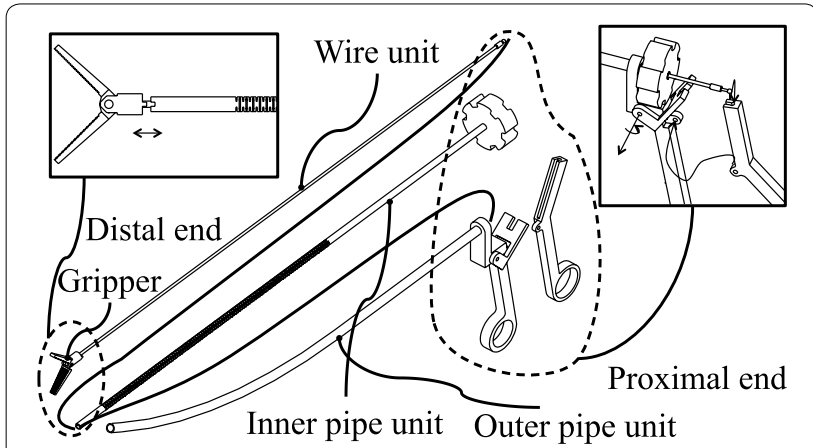

Fig. 13 The structure of an easy-to-disassemble forceps

pipe has high flexural rigidity and cannot pass through the curved portion of the outer pipe. The assembly procedure is the reverse of the disassembly procedure.

\section{Conclusion}

To improve the manipulability of arc-shaped forceps, this paper discusses the method of the design of the shaft axis. Usually, concentric pipe structures are used to transmit the rotational motion of the proximal end to the distal end of the gripper. In this method, the shape of the bent inner pipe is different from that of the outer pipe. This causes strong contact between the outer and inner pipes if the inner pipe has strong flexural rigidity. This causes a large friction torque and a large angle difference (twist angle) between the distal end and the proximal end of the inner pipe. A low friction and elastic plastic-tube for the inner pipe would be valid to reduce the required torque. However, the Young's modulus of plastic is significantly lower than that of metal and the torsional rigidity is also low, which results in a large twist angle. This paper proposes a procedure to fit the bent inner pipe to the outer pipe such that only the ends of the pipes are in contact with each other. The inner pipe with slits adjusts its flexural rigidity. The proposed method can be applied to various curves.

The following procedures could produce the optimized slit design.

- Different slit intervals and width change the flexural rigidity of the inner pipe. First, the database of the equivalent flexural rigidity is developed. Repeat the following by changing the contact force between the inner and outer pipes.

- The desired distribution of the flexural rigidity of the inner pipe is calculated from the outer pipe curve. 
- It is discretized by applying the database.

- The candidate is excluded if the stress and error are larger than the allowable values.

- Slit pattern of the minimum twist angle is selected among the candidates.

We performed simulations and several experiments and confirmed the following. The twist angle was measured when a load torque of $20 \mathrm{Nmm}$ was applied.

- In the $8 \mathrm{~mm}$ forceps (Ti-6Al-4V) simulation, the twist angle was $4.30^{\circ}$.

- In the $5 \mathrm{~mm}$ forceps (SUS630) simulation, the twist angle was $2.93^{\circ}$, whereas the twist angle of the PEEK inner pipe was $24.3^{\circ}$.

- In the $5 \mathrm{~mm}$ forceps experiments, SUS304 was selected, which has a similar Young's modulus to SUS630. The twist angle of $2.8^{\circ}$ was consistent with the simulation result.

- In the ex vivo experiments, the forceps were inserted into the chicken liver, minced pork meat, and porcine blood the rotation torque was measured. It changed from 5.1 to 5.3, 5.4, and 3.8 Nmm respectively. Moreover, it was $5.3 \mathrm{Nmm}$ when blood was poured in the outer pipe and coagulated. These increase are small.

Therefore, we confirmed that the proposed method can minimize the twist angle and reduce the required torque. Although a PTFE coating is valid to reduce friction, this method is effective in terms of wear resistance. Moreover, we consider sterilization by an easy-to-disassemble forceps structure. In vivo experiments are required to test the developed forceps under more rigorous conditions, which is our future work.

\section{Authors' contributions \\ All authors equally contributed. All authors read and approved the final manuscript.}

\footnotetext{
Author details

1 Department of Mechano-Micro Engineering, Tokyo Institute of Technology, 4259, Nagatsuta-cho, Midori-ku, Yokohama, Kanagawa 226-8502, Japan. ${ }^{2}$ Department of Mechanical Engineering, School of Engineering, Tokyo Institute of Technology, 4259, Nagatsuta-cho, Midori-ku, Yokohama, Kanagawa 226-8502, Japan.
}

\section{Competing interests}

The authors declare that they have no competing interests.
Received: 20 August 2016 Accepted: 3 March 2017

Published online: 31 March 2017

\section{References}

1. Kumagai T, Yamashita J, Morikawa O, Yokoyama K, Fujimaki S, Konishi T, Ishimasa H, Murata H, Tomoda K (2008) Distance education system for teaching manual skills in endoscopic paranasal sinus surgery using "hypermirror" telecommunication interface. In: 2008 IEEE virtual reality conference, pp 233-236. doi:10.1109/VR.2008.4480779

2. Podolsky ER, Curcillo PG II (2010) Reduced-port surgery: preservation of the critical view in single-port-access cholecystectomy. Surg Endosc 24(12):3038-3043

3. Sugimoto T, Kiyokawa Y, Kishikawa M, Kishimoto S, Nomura F, Tokumaru T, Kawada K, Okada T, Ariizumi Y (2012) A Discussion of the Necessity of Improved Surgical linstruments used in ELPS (Eendoscopic LatyngoPpharyngeal Surgery). Oto Rhino Laryngol Tokyo 55(5):369-374 (in Japanese)

4. Lin F-Y, Bergeles C, Yang G-Z (2015) Biometry-based concentric tubes robot for vitreoretinal surgery. In: 2015 37th annual international conference of the IEEE Engineering in Medicine and Biology Society (EMBC), IEEE, New York, pp 5280-5284

5. Dupont PE, Lock J, Itkowitz B, Butler E (2010) Design and control of concentric-tube robots. IEEE Trans Robot 26(2):209-225

6. Steger JR, Gerbi CR, Prisco GM, Rogers TW (2013) Curved cannula instrument. U.S. patent US8551115B2

7. Kroh M, El-Hayek K, Rosenblatt S, Chand B, Escobar P, Kaouk J, Chalikonda S (2011) First human surgery with a novel single-port robotic system: cholecystectomy using the da vinci single-site platform. Surg Endosc 25(11):3566-3573

8. Kallidonis P, Kontogiannis S, Kyriazis I, Georgiopoulos I, Al-Aown A, Stolzenburg J-U, Liatsikos E (2013) Laparoendoscopic single-site surgery in kidney surgery: clinical experience and future perspectives. Curr Urol Rep 14(5):496-505

9. ADACHI INDUSTRY CO., L (2014) Arc-shaped forceps for endoscopic surgery. Japan patent JP2014-23922A (in Japanese)

10. Tadano K, Sumino W, Kawashima K (2009) Development of pneumatically driven forceps manipulator with force display. J Robot Soc Japan 27(5):538-545 (in Japanese)

11. Kim J-S, Lee D-Y, Kim K, Kang S, Cho K-J (2014) Toward a solution to the snapping problem in a concentric-tube continuum robot: Grooved tubes with anisotropy. In: 2014 IEEE international conference on robotics and automation (ICRA). IEEE, New York, pp 5871-5876

12. Peirs J, Van Brussel H, Reynaerts D, De Gersem G (2002) A flexible distal tip with two degrees of freedom for enhanced dexterity in endoscopic robot surgery. In: Proceedings of the 13th micromechanics Europe workshop, pp 271-274

13. The Bent Forceps of ADACHI INDUSTRY. http://www.adachikk.co.jp/ contents/products/download/tanko.pdf (in Japanese)

14. Biswas S, Vijayan K (1992) Friction and wear of ptfe-a review. Wear 158(1-2):193-211

15. Ryan P (1999) Surgical blade coatings. Google Patents. US Patent App. $09 / 356,228$ 